\title{
Zur Gestaltung des digitalen Wandels jetzt in Bibliotheken investieren
}

\author{
Aktueller „Bericht zur Lage der Bibliotheken 2018/19“ \\ erschienen
}

http://doi.org/10.1515/bd-2019-0003

Parallel zu der Entwicklung der digitalen Revolution ist auch das Medien- und Serviceangebot der Bibliotheken im Umbruch: die Bereitstellung von sowohl analogen als auch digitalen Medien und Dienstleistungen ist eine entscheidende Herausforderung für die Zukunftsfähigkeit der Bibliotheken. Hierfür sind erhebliche finanzielle Aufwendungen für Investitionen und den laufenden Betrieb notwendig. Für die innovationsorientierte Weiterentwicklung zeitgemäßer Bibliotheksangebote in den Kommunen fordert der Deutsche Bibliotheksverband daher eine deutlich bessere und langfristig zuverlässige Finanzausstattung. Diese ist dringend notwendig, zeigt doch die aktuelle Umfrage des Verbandes zu der Finanzsituation von rund 1.350 Bibliotheken, dass für dieses deutlich erweiterte Aufgabenspektrum keine zusätzlichen Mittel bereit stehen.

Eine Stagnation in der Finanzausstattung von Bibliotheken kommt realen Kürzungen gleich, übernehmen Bibliotheken doch auch eine Vielzahl an zusätzlichen, gesellschaftlich relevanten Aufgaben, beispielsweise in den Bereichen kulturelle und digitale Bildung, Integration und Inklusion.

„Um den Zugang zum Wissen unserer Welt zu ermöglichen und die Teilhabe aller Bürgerinnen und Bürger am digitalen Wandel als ihre Kernaufgabe gut zu bewältigen, brauchen Öffentliche und Wissenschaftliche Bibliotheken politische Unterstützung, verlässliche Rahmenbedingungen, eine solide Finanzierung als Voraussetzungen für ihre Entwicklungsmöglichkeiten." So Barbara Lison, Bundesvorsitzende des Bibliotheksverbandes. „Weiterhin zentral für die zukünftigen Dienstleistungen in den Bibliotheken ist ein zeitgemäßes internationales, europäisches und deutsches Urheberrecht, das Lehre und Forschung in der digitalen Welt fördert und den Öffentlichen Bibliotheken ermöglicht, E-Books genauso zu verleihen wie gedruckte Bücher.“ So Lison weiter.

Mit dem seit 2010 jährlich veröffentlichten „Bericht zur Lage der Bibliotheken“ informiert der Deutsche Bibliotheksverband sowohl politische Entscheidungsträger als auch die Öffentlichkeit über die Leistungen und die Arbeitsbedingungen der Bibliotheken und formuliert entsprechende bibliothekspolitische Forderungen. In seiner aktuellen Ausgabe informiert der Bericht über die Forderungen nach einer Sonntagsöffnung in Öffentlichen Bibliotheken, nach Digital- 
strategien in den Kommunen für die Bibliotheken und nach einem zeitgemäßen Urheberrecht. Akzentuiert werden in dem Bericht außerdem die Aufgaben der Provenienzforschung und das Potenzial von Bibliotheken für die Erreichung der Nachhaltigkeitsziele der UN-Agenda 2030.

Zentrales Anliegen des Deutschen Bibliotheksverbandes ist es, allen Bürgerinnen und Bürgern den bestmöglichen Zugang zu zeitgemäßen Bibliotheksangeboten zu verschaffen. Mit diesem Ziel setzt er sich für die Sicherung und Optimierung des rechtlichen und finanziellen Rahmens für die Arbeit von Bibliotheken ein.

\section{Deutscher Bibliotheksverband e.V.}

Maiken Hagemeister, Pressesprecherin und Leitung Kommunikation, Tel.: 0 30/644 989925

E-Mail: hagemeister@bibliotheksverband.de, http://www.bibliotheksverband.de, http://www.bibliotheksportal.de 